\title{
Enlarged lymph nodes diagnosed with Langerhans cell histiocytosis found in a direct inguinal hernia: a case report
}

\author{
Jong Hyuk Yun ${ }^{1}$, Myoung Won Son ${ }^{1}$, Geum Jong Song ${ }^{1}$, Hye In Ahn², Sang Ho Bae ${ }^{1}$, Moon Soo Lee \\ ${ }^{1}$ Department of Surgery, Soonchunhyang University Cheonan Hospital, Cheonan, Korea \\ ${ }^{2}$ Department of Pathology, Soonchunhyang University Cheonan Hospital, Cheonan, Korea
}

\begin{abstract}
It is unusual that an unexpected mass is encountered within a hernia sac. This report describes a patient diagnosed with Langerhans cell histiocytosis (LCH) after surgery for an inguinal hernia. A 64-year-old male patient presented with inguinal mass over a 1-year period. Direct inguinal hernias were found in both sides, and enlarged lymph nodes were found in both hernia sacs. Laparoscopic totally extraperitoneal repair was done, and one enlarged lymph node within inguinal hernia sac was excised for diagnostic purposes. Microscopic findings showed the distinctive cytologic features of Langerhans cells and immunohistochemical staining are positive for CDla and S-100. LCH is a rare disorder, and the involvement of the lymph nodes with no other sites of disease is uncommon. To the best of our knowledge, this is the first report of LCH within an inguinal hernia sac. Multidisciplinary approach should be considered to provide better detection and treatment.
\end{abstract}

Keywords: Langerhans cells, Histiocytosis, Inguinal hernia, Lymph nodes
Received October 11, 2021

Revised 1st November 26, 2021

2nd December 3, 2021

Accepted December 4, 2021

Corresponding author

Myoung Won Son

Department of Surgery, Soonchunhyang University Cheonan Hospital, 31

Suncheonhyang 6-gil, Dongnam-gu,

Cheonan 31151, Korea

Tel: +82-41-570-3636

Fax: +82-41-571-0129

E-mail: mwson@schmc.ac.kr ORCID:

https://orcid.org/0000-0001-5255-9234

Copyright (๑) The Korean Society of Endoscopic and Laparoscopic Surgeons.
This is an Open Access article distributed under the terms of the Creative Commons Attribution Non-Commercial License (http:// creativecommons.org/licenses/by-nc/4.0/) which permits unrestricted non-commercial use, distribution, and reproduction in any medium, provided the original work is properly cited.

\section{INTRODUCTION}

The repair of inguinal hernias is one of the most common surgeries worldwide. An inguinal hernia is considered to be a benign condition, and repairs without recurrence are possible in nearly 95\% of cases [1]. Despite the high incidence and resolution rates of this condition, unusual cases do occur, such as those where there is an unexpected mass found within the hernia. This report describes a patient diagnosed with Langerhans cell histiocytosis (LCH) after surgery for an inguinal hernia. To the best of our knowledge, this is the first report of LCH within a direct inguinal hernia sac.

\section{CASE REPORT}

A 64-year-old male patient presented with a painless, non-tender, reducible, bilateral inguinal mass over a 1-year period. An ultrasound scan revealed an inguinal hernia with an enlarged inguinal lymph node, and computed tomography (CT) imaging revealed enlarged inguinal and external iliac lymph nodes; in light of these findings, lymphoma was suspected (Fig. 1). Laparoscopic totally extraperitoneal repair with tissue biopsy was planned in order to confirm the diagnosis. Three infraumbilical ports were used, one camera port and two working ports. We used balloon dissector (Spacemaker; Medtronic, Dublin, Ireland) to prepare the extraperitoneal space. Direct inguinal hernias were found on 


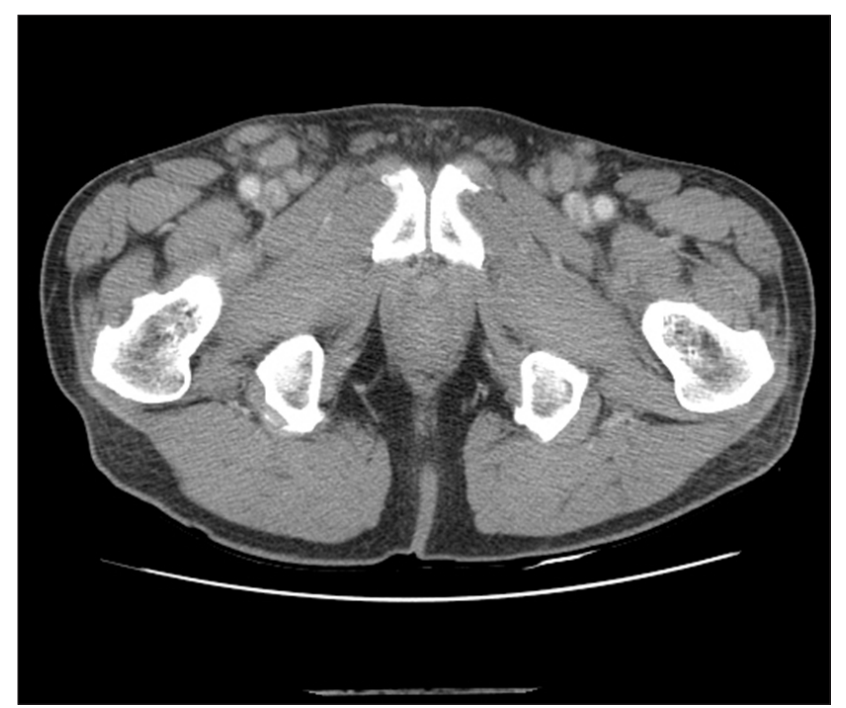

Fig. 1. Initial computed tomography scan was notable for multiple enlarged lymph nodes. both sides that bulged medial to the inferior epigastric vessels. Enlarged lymph nodes were found in both the direct hernia sac along with one enlarged lymph node within the right direct inguinal hernia sac, which was excised for diagnostic purposes (Fig. 2). The Parietex hydrophilic anatomical meshs (Medtronic) were used in both sides. The operative time was 40 minutes, and estimated blood loss was $20 \mathrm{~mL}$. He had an uneventful postoperative recovery course and was discharged on the first postoperative day. Hematoxylin and eosin stain with a low power view showed the connective tissue of the hernia sac with enlarged lymph nodes. Microscopic findings of the lymph node biopsy showed sinusoidal and paracortical infiltration of tumor cells with preservation of some lymphoid follicles; the tumor cells showed the distinctive cytologic features known for Langerhans cells (LCs), with irregular and elongated nuclei with prominent nuclear grooves and folds (Fig. 3). Immunohistochemical staining of CDla and S-100 indicated strong and diffuse expression in tumor cells, and combined with the aforementioned findings, the diagnosis of LCH was confirmed (Fig. 4). The patient was transferred
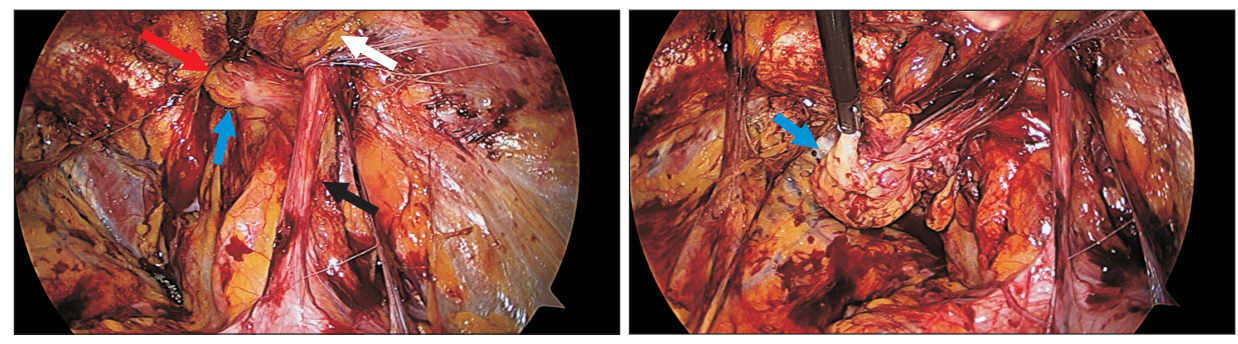

Fig. 2. Enlarged lymph node was observed within direct hernia defect and excised. Red arrow, direct hernia defect; blue arrows, mass; black arrow, vas deferens; white arrow, inferior epigastric vessels.
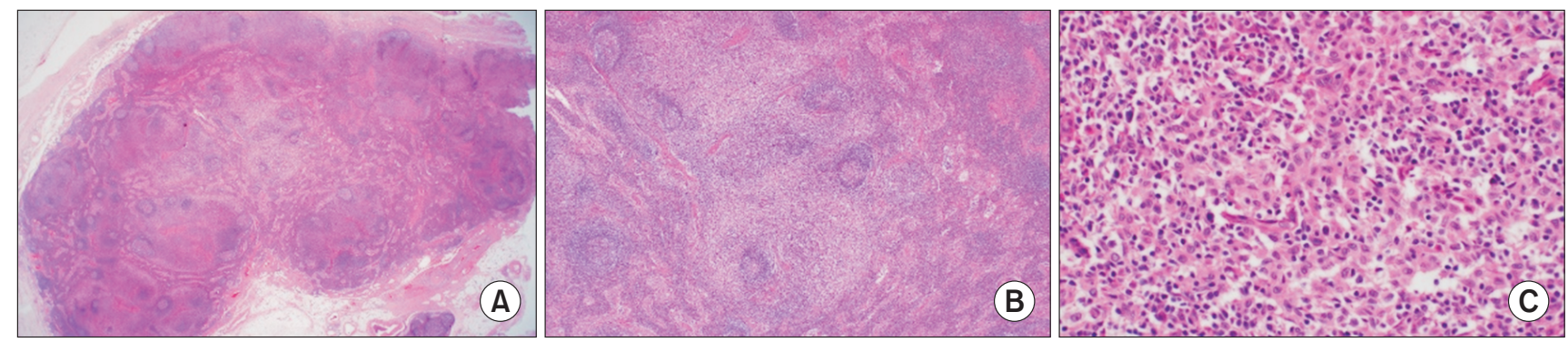

Fig. 3. (A) Hernia sac connective tissue with enlarged lymph node (H\&E stain, $\times 12.5)$. (B) Sinusoidal and paracortical infiltrate of tumor cells with preservation of some lymphoid follicles (H\&E stain, $\times 40)$. (C) Irregular and elongated nuclei with prominent nuclear grooves and folds $(H \& E$ stain, $\times 400)$.

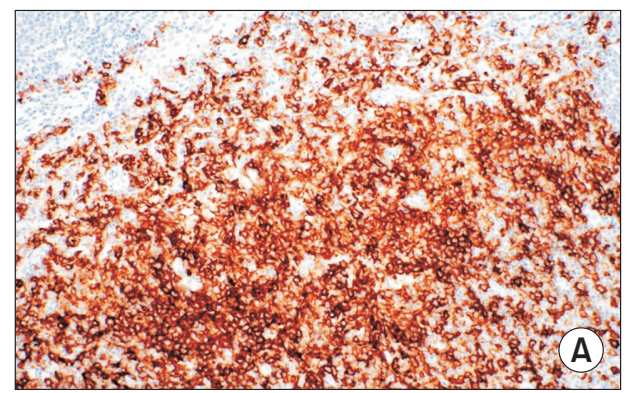

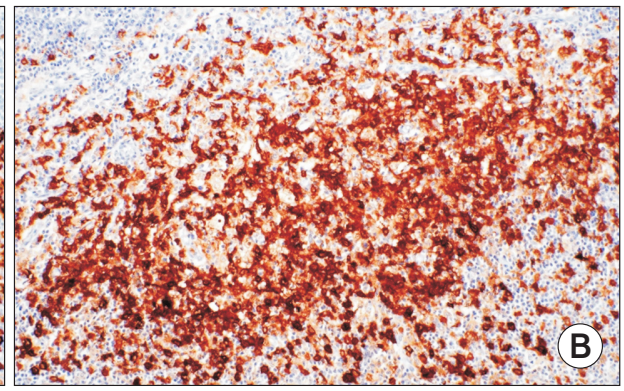

Fig. 4. Immunohistochemical staining $(\times 200)$ of CD1a (A) and S-100 (B). The images show strong and diffuse expression in tumor cells. 

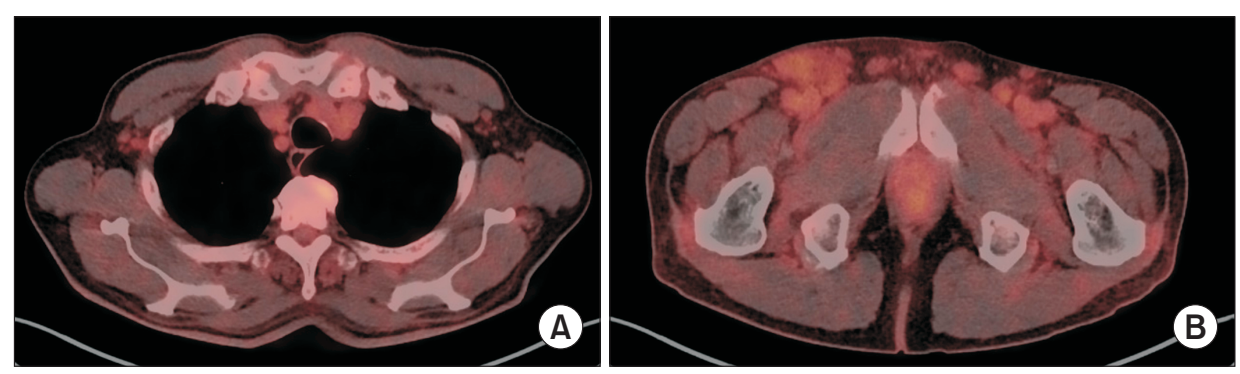

Fig. 5. Fluorodeoxyglucose (FDG) positron emission tomography scan showed mild to moderate FDG uptake of enlarged lymph nodes in bilateral axillary areas (A), external iliac and inguinal areas (B).

to the division of hematology-oncology in order for a treatment plan to be developed. Chest and abdominal CT, as well as fluorodeoxyglucose positron emission tomography-CT, were performed for a clinical workup. Multiple lymph node enlargements were found in the bilateral external iliac areas, inguinal area, and bilateral axillary areas, but there were no suspicious lesions of LCH in other organs (Fig. 5). Chemotherapy (vinblastine and prednisone) was recommended, and a test for the BRAF-V600E mutation was planned; however, the patient was transferred to another hospital for personal reasons and therefore could not be followed up with further.

\section{DISCUSSION}

Despite the high incidence of inguinal hernia, it is very rare to find a mass in inguinal hernia sac. These masses can be made up of normal structures including the appendix, ovary, and bladder; however, cord lipoma, varicocele, desmoid tumor, primary neoplasm including liposarcoma, lymphoma, testicular carcinoma, and metastases from other primary tumors can also be found [2]. Tumors presenting in the inguinal hernia sac are considered extremely rare; less than $0.5 \%$ of hernias contain primary or metastatic tumors, and it is more common that neoplasms metastasizing from the gastrointestinal tract, ovary, and prostate are found [3]. So far, there have been no reports of LCs found in inguinal hernia sac.

LCs are dendritic cells that normally reside in the skin and mucosa, and are the primary antigen-presenting cells of the skin. After LCs encounter antigens, they migrate to lymph nodes and present the antigens they have encountered to T cells [4]. Histiocytosis is a rare disorder that is characterized by the accumulation of cells thought to be derived from dendritic cells or macrophages. $\mathrm{LCH}$, which is the most common histiocytic disorder, encompasses conditions characterized by the aberrant function, differentiation, or proliferation of the cells of the mononuclear phagocyte system [5]. The disorder is characterized by granulomatous lesions composed of clonal pathologic "histiocytes" (CD207+ cells), and is historically poorly understood [6]. Recently, LCH has been defined as a myeloid neoplasm, not an immune disorder, based on various biological evidence that has been un- covered $[5,7,8]$.

LHC is a rare disorder that occurs at all ages, but it predominantly affects children; four to eight children per million and one to two adults per million are affected each year [5,6]. Most adult LCH cases involve only a single organ system, such as the bones or lungs [8], and the clinical features of adult LCH are poorly understood because of the rarity of adult cases.

LCH has variable clinical manifestations, ranging from a single indolent disease to a systemic and aggressive disease course that can lead to death and wide-ranging organ involvement $[5,7]$. The disease can be categorized as either single-system LCH with multifocal or unifocal involvement, or multisystem LCH with multiple organ involvement [7]. Granulomatous lesions and inflammatory infiltrates can arise in any organ, but are particularly prevalent in bone, skin, lungs, and the pituitary [5]; the most common site of involvement is bone, followed by skin and granulomatous lesions in the scalp, mucosal lesions of the oral cavity and genitals, and cystic lung lesions. These lesions may or may not be painful, and may be found incidentally [9]. Lymph node involvement has also been reported, usually occurring in pediatric patients with known systemic $\mathrm{LCH}$, though primary $\mathrm{LCH}$ of lymph nodes without involvement of other sites is rare [10]. In this case, enlarged lymph nodes in the direct inguinal hernia sac were found and excised to test to confirm the correct diagnosis. To our knowledge, a finding of $\mathrm{LCH}$ within the hernia sac has never before been reported.

The diagnosis of LCH is based on clinical and radiological findings in combination with histopathological and immunophenotypic analyses. Formal diagnosis of $\mathrm{LCH}$ requires a tissue biopsy for histologic evaluation to identify tissue infiltration by LCs [4,9]. Neoplastic LCs are uniformly positive for CD1a, CD207, and S100, and positive test results for three proteins are mandatory for the confirmation of diagnosis [4].

The histopathologic differential diagnosis of $\mathrm{LCH}$ presenting with lymphadenopathy includes all conditions that may mimic LCH depending on the extent of nodal involvement by the disease, such as Kimura's disease, dermatopathic lymphadenitis, and Hodgkin's lymphoma [10]. There are no standard therapies for adult $\mathrm{LCH}$, and treatment options are based on organ involvement and the extent of the disease [7]. Local therapies are only 
recommended for limited cases such as single-system LCH with isolated skin or bone involvement; systemic therapy is strongly recommended for most cases [5,7]. Since only multiple nodal involvements were found in this case, it would have been appropriate to implement systemic therapy rather than radiation therapy. For multiple lymph nodal involvements in pediatric LCH, the combination of vinblastine and prednisolone could serve as the standard course of therapy, but adult patients who undergo combination therapy tend to show higher toxicity, resulting in a poorer overall response [7]. Since the discovery of the BRAFV600E mutation associated with LCH, there have been many advances in the molecular pathophysiology of the disease. These results have made the application of targeted therapy possible, although further investigation is needed [7]. Overall, the treatment of LCH remains challenging, particularly in adults.

In conclusion, $\mathrm{LCH}$ is a rare disorder, and the involvement of the lymph nodes with no other sites of disease is uncommon. Lymph node involvement in LCH within the hernia sac has not been reported before the case discussed here. Tumors presenting within the hernia sac are considered to be extremely rare and should be managed with caution. It is unusual an unexpected mass is encountered within a hernia sac, even for a skilled hernia surgeon. In the case of laparoscopic total extraperitoneal hernia repair, tissue biopsy of unexpected findings within the hernia sac may often be neglected. However, it is important to be prepared to conduct tissue biopsy when necessary in these cases in order to make an accurate diagnosis, as early recognition of rare disorders such as LCH is essential for proper treatment. A proper preoperative clinical work-up and management plan should be performed, and a multidisciplinary approach involving surgeons, pathologists, radiologists, and hemato-oncologists should be considered to provide better detection and treatment for rare disorders such as this one.

\section{NOTES}

\section{Ethical statement}

This study was approved by the Institutional Review Board of Soonchunhyang Cheonan Hospital with a waiver of informed consent (No. SCHCA 2021-03-034).

\section{Author's contributions}

Conceptualization, Data curation: MWS, JHY

Formal analysis: JHY, GJS, SHB

Investigation, Methodology: JHY, HIA

Project administration: MWS, MSL

Visualization: HIA, GJS, SHB
Writing-review \& editing: JHY, MWS, MSL

All authors read and approved the final manuscript.

\section{Conflict of interest}

All authors have no conflict of interest to declare.

\section{Funding/support}

This work was supported by the Soonchunhyang University Research Fund.

\section{ORCID}

Jong Hyuk Yun, https://orcid.org/0000-0002-8746-4945

Myoung Won Son, https://orcid.org/0000-0001-5255-9234

Geum Jong Song, https://orcid.org/0000-0002-1067-8099

Hye In Ahn, https://orcid.org/0000-0001-9221-672X

Sang Ho Bae, https://orcid.org/0000-0003-1138-0013

Moon Soo Lee, https://orcid.org/0000-0002-3955-1331

\section{REFERENCES}

1. Kulacoglu H, Köckerling F. Hernia and cancer: the points where the roads intersect. Front Surg 2019;6:19.

2. Bhosale PR, Patnana M, Viswanathan C, Szklaruk J. The inguinal canal: anatomy and imaging features of common and uncommon masses. Radiographics 2008;28:819-913.

3. Nicholson CP, Donohue JH, Thompson GB, Lewis JE. A study of metastatic cancer found during inguinal hernia repair. Cancer 1992; 69:3008-3011.

4. Ungari M, Ferrero G, Varotti E, et al. Langerhans cell histiocytosis of an intra-mammary lymph node in an 18-year-old woman. Pathologica 2020;112:50-55.

5. Allen CE, Merad M, McClain KL. Langerhans-cell histiocytosis. N Engl J Med 2018;379:856-868.

6. Zinn DJ, Chakraborty R, Allen CE. Langerhans cell histiocytosis: emerging insights and clinical implications. Oncology (Williston Park) 2016;30:122-139.

7. Kobayashi M, Tojo A. Langerhans cell histiocytosis in adults: advances in pathophysiology and treatment. Cancer Sci 2018;109:3707-3713.

8. Zhu H, Ma Y, Sun L, Zhang R, Lv L, Wang A. LANGERHANS cell histiocytosis with lymph node involvement presenting as erythroderma. Acta Derm Venereol 2019;99:99-100.

9. DiCaprio MR, Roberts TT. Diagnosis and management of langerhans cell histiocytosis. J Am Acad Orthop Surg. 2014;22:643-652.

10. Edelweiss M, Medeiros LJ, Suster S, Moran CA. Lymph node involvement by Langerhans cell histiocytosis: a clinicopathologic and immunohistochemical study of 20 cases. Hum Pathol 2007;38:1463-1469. 